DOI: https://doi.org/10.47405/aswj.v6i4.172

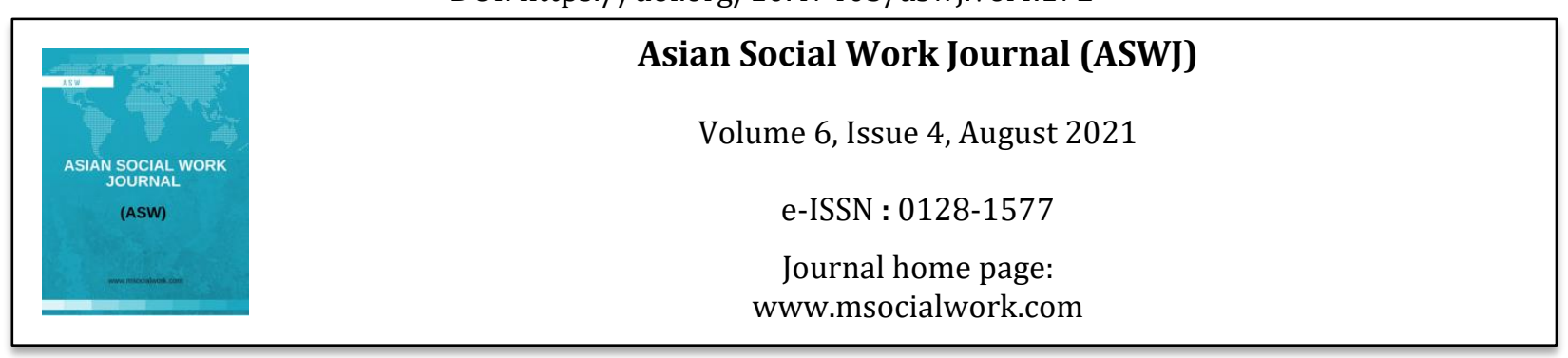

\title{
Feasibility of a Qualitative Study Exploring the Experience of Deaf Individuals from Malaysia Studying Abroad: A Preliminary Study
}

\author{
NurFarhana A. Aftar ${ }^{1}$ \\ 1Faculty of Psychology and Education, Universiti Malaysia Sabah (UMS) \\ Correspondence: NurFarhana A. Aftar (farhana@ums.edu.my)
}

\begin{abstract}
This is a feasibility qualitative study involving a triangulation data collection method. The aim of the study is to develop a qualitative research to explore the lived experience of deaf individuals in attempt to view and understand their experience from their perspectives. This preliminary study involved a semi-structured interview with one deaf participant, observations of short video clips, and document review of relevant blog entries by a deaf individual from Malaysia. Thematic analysis was completed, and six emerging themes found were access, communication/interaction, gaining perspectives, interpreters, optimistic, and social support. As this was a feasibility study, modifications were made throughout the study. Limitations of this study was documented and taken into consideration in designing further studies.
\end{abstract}

Keywords: Deaf peoples, access, interaction, interpreter, optimistic, social support

\section{Introduction}

This is a feasibility study in designing a qualitative research focusing on exploring the experience of deaf individuals who were originally from Malaysia and then moved to the United States. Through this study, the researcher attempted to design and assess the feasibility of a qualitative study aimed at exploring the participants' personal experiences in both Malaysia and the United States. The goal of the research was to identify potential areas of needs based on insights obtained from the participants' experience and perspectives. The qualitative approach aims to explore a topic rather than to conclude on how a topic fit into any specific existing theoretical framework. Therefore, in this study, there is no specific theoretical framework that was used to guide the directions of the research. The exploration was however conducted based on the framework of the constructivist paradigm. Based on the basic assumption of the constructivist paradigm, the researcher aimed to gather data by attempting to understand the complexity of this experience from the viewpoint of those who lived through it. This study was designed as a preliminary study to determine the feasibility of this research approach. Therefore, the following literature review focused on designing the research methodology of this exploration.

\section{Literature Review}

The phenomenon that was aimed at for this research was the life experience of deaf individuals who originated from Malaysia and then moved to the United States. A major underlying assumption of this 
research idea is that this phenomenon is a life changing phenomenon in terms of how individuals view themselves and the world around them. Interestingly, this assumption is similar to the findings of De Clerck (2007). In addition, it is also assumed that there are differences in terms of insights and services that are related to the deaf community between Malaysia and the United States. Consequently, this would presumably affect the development of deaf individuals' perspectives in life. To illustrate and support this assumption, the researcher referred to a research by Wathum-Ocama and Rose (2002) that specifically focused on immigrants' views on the education of their deaf children. Finally, in developing the methodology, the researcher referred to a research by Xue (2013) which was conducted on a similar phenomenon. These literatures were reviewed and referred to in the development of this current research.

\section{The Lived Experience as a Phenomenon}

An exploratory case study by De Clerck (2007) was analyzed to support the assumption for this research. De Clerck's (2007) research was conducted on a small sample of Flemish deaf participants. As a prerequisite for participation in the study, the participants needed to have had the experience of encountering deaf culture that was a turning point in their lives. The inclusion criteria included whether the participant has encountered deaf culture that was a turning point in their lives, which is very similar to the phenomenon that this current research is investigating. De Clerk (2007) conducted member checking and peer debriefing as their criteria for rigor.

As a result of this qualitative study, De Clerk (2007) developed the circle of deaf empowerment as a model to demonstrate what the participants described as stages of 'sleeping' and 'waking up'. The participants who had been exposed to barrier-free deaf environments described that prior to the exposure, they were in the 'sleeping' stage which in general was confined to the prevalence of oralism and based on the expectations of the majority 'hearing' culture. Upon exposure to barrier-free deaf environments they experience the 'waking-up' stage and this is when they become aware of a different way of viewing deafness. Having the experience of being in a deaf culture abroad, made them 'wakeup' and realize that it has been proven that deaf people can do a lot of things, they have their own natural language and their own culture. Overall, the findings suggest that encountering barrier-free deaf environments were indeed a turning point in their lives (De Clerck, 2007).

\section{The Assumption of the Lived Experience}

One assumption that is involved in this current research is the assumption that there are differences in terms of personal insights and availability of services available for the deaf community between Malaysia and the United States, which in turn would affect an individual's perspectives to life. Wathum-Ocama and Rose (2002) conducted a research on education that can illustrate how immigrants have their own ways of conceptualizing or understanding education for their deaf children. The focus of the research by Wathum-Ocama and Rose (2002) were the attitudes, perceptions, and feelings of Hmong parents of deaf or hard of hearing child attending a U.S. public school. Hmong immigrants are people who originated from Laos, which is in the Southeast Asia, and they were the targeted population for the research. This is because as immigrants they are exposed to environments that are almost entirely alien such as the public school system, which can especially be perplexing when a deaf child is involved (Wathum-Ocama \& Rose, 2002). Wathum-Ocama and Rose's (2002) research was conducted qualitatively, and data collection was conducted via semi-structured interviews, examination of students' records, and observation of the participants, also known as triangulation, to support the internal validity of the study.

\section{The Qualitative Phenomenological Approach}

In developing the methodology of this current research, a study by Xue (2013) was used as a reference. Xue (2013) conducted a qualitative study involving Chinese international graduate students' experiences with group work. This was particularly relevant to this current research in a sense that the participants were those who had been exposed to a situation in two different countries, in which one was the United States. Xue (2013) used a qualitative phenomenological approach to obtain an in-depth 
Asian Social Work Journal (ASWJ), Volume 6, Issue 4, (page 1 - 9), 2021

DOI: https://doi.org/10.47405/aswj.v6i4.172

understanding for the participants' experiences with group work. The researcher performed purposeful sampling and recruited participants who fulfilled the inclusion criteria. The inclusion criteria included Chinese students who were not English native speakers, who has had bachelor's degrees from Chinese institutions of higher education and had studied for master or doctoral degrees at the researcher's university in the United States for more than one year. Data collection was conducted by face-to-face semi structured interviews. The interviews were tape recorded and data were transcribed verbatim by the researcher. To establish criteria for rigor, the researcher conducted peer debriefing and member checking (Xue, 2013).

\section{Methodology}

This study was designed based on a qualitative phenomenological approach to obtain an in-depth insight of the participants' experience. A phenomenological approach was chosen for this study because the focus was on a group of people who have a common experience among them and not everyone has had the same experience. The focus of this research was on the participant's lived experience (Schwandt, 2000). It involved exploring the meaning of their experience, with an assumption that there is an essence to that experience that is shared among the participants. All theories, hypotheses or explanations are set aside and was not used as the basis for this research. Everything within a phenomenological study is about what the experience was and how it was experienced (Wertz, 2011).

\section{Researcher as Instrument: Philosophical Positioning}

As an individual who originated from Malaysia and grew up with a deaf sibling, the current researcher has existing opinions and views about being deaf in Malaysia. This includes the need to bring attention to areas of needs for deaf community in Malaysia to ultimately create a space with reduced barriers. However, it is acknowledged that the researcher is not a member of the deaf community; therefore, the researcher's perspectives may not represent the perspectives of individuals from this community.

At the time that the research was conducted, the researcher was based in the United States. The researcher has the experience of attending an institution that offers a reduced barrier environment for deaf individuals which had been an eye-opening experience. The experience changed the researcher's perspectives with regards to deafness and the world surrounding the researcher. Therefore, it was assumed that deaf individuals who were originally from Malaysia would also experience a life changing phenomenon upon exposure to a similar environment. Given the researcher's background, the constructivist paradigm was deemed a good fit for the current study.

Viewing research from the constructivist paradigm's standpoint means to have an axiology that involves balancing the views of everyone involved in the research (Mertens, 2010; Guba \& Lincoln, 1989). As a researcher, the researcher can personally relate to this topic and has existing perspectives on the topic, but the researcher does not represent the targeted community that is being addressed. Therefore, by conducting this research the researcher is using existing perspective to gather data from a different standpoint. Ultimately, the outcome of this research is the combination of ideas from everyone who were involved. By addressing specific research questions through this study, the researcher brings up issues that the participants may not realize, even if they had experienced these themselves. This is aligned with the constructivist's axiology, which is to make participants aware of their experience by organizing this experience into a more structured form of information. Overall, as a constructivist, it is important that this research process involves good rapport between the researcher and participants, as well as reciprocity, where both the researcher and participants play roles that are of equal importance. The outcome of this research would hopefully be able to reveal the reality they had experience with regards to differences between the deaf experience in Malaysia and the United States.

The ontological assumption of the constructivist paradigm is that there are multiple realities, which are socially constructed (Mertens, 2010). By taking this perspective the researcher believes the reality that exists out there had been created by the society based on social norms. Considering that the deaf 


\section{Asian Social Work Journal (ASWJ), Volume 6, Issue 4, (page 1 - 9), 2021 \\ DOI: https://doi.org/10.47405/aswj.v6i4.172}

population is a minority group, this means that the reality with regards to the world of deaf individuals we currently have are based on what the society has constructed which would less likely lean towards the advantage of the deaf community themselves. At the same time, the researcher acknowledges that there can be multiple realities depending on which group of population that constructed them. Therefore, by conducting this research the researcher hoped to construct a reality based on the perspective of individuals from the targeted population which is the deaf community. Following the outcome of this research, future work will be directed towards incorporating this newfound reality into the reality that currently exists. This means finding a way to gradually and effectively merge the realities that we now have.

The epistemology of a constructivist's research involves the interaction between the researcher and participants (Mertens, 2010). Therefore, the interaction and the relationship between the researcher and the participants are critical in gathering this information. The finding of this research is dependent on everything that is shared between the researcher and the participants. This involves that the researcher's perspectives and experience with regards to the topic of discussion is made explicit to the participant. At the same time, the same things are expected from the participants. Making the researchers' viewpoints explicit to the participants, will guide the participants to organize their experience and perspectives in a similar way. Ultimately, the outcome of this research will be based on the direction that this interaction takes place.

\section{Sampling}

A snowball sampling was planned for this research by identifying a key person from the international office for assistance with recruitment for participants. A general email was sent out to all students and alumni who were known to originate from Malaysia. However, no volunteer had come forward through this method. The method of participant recruitment was revised, and an advertisement was then posted on an online social network website. Several people responded to the advertisement and eventually one had agreed to participate in the study.

The inclusion criteria for this study were that i) the person was originally from Malaysia, and has had the experience of attending school or working in Malaysia, ii) the person has moved to the US for at least 1 year, and has had the experience of going to school or working in the US and iii) the person is a deaf or hard of hearing individual above the age of 18 years old.

\section{Data Collection Methods}

Data collection included a semi-structured interview, an observation, and a document review. Some changes were made due to limitations that were faced during this study.

\section{Semi-structured Interview}

A face-to-face session was not possible due to time and financial factors; therefore the interview was conducted through a video call by using Skype. An initial Skype session was conducted with the participant for 20 minutes to explain about the study in detail, and provide the participant the opportunity to ask questions, before agreeing to participate. Documents related to informed consent, release of video, and agreement to changes were password protected and sent as an attachment through communication via email. The interview was conducted via Skype for one hour. This was recorded using an extension software for recording Skype (Evaer), and the laptop used for this purpose was ensured to be password protected for data protection.

The interview was conducted in American Sign Language. Data were transcribed verbatim after the interview and any ambiguity that appeared during the process of transcribing were clarified further with the participant. Follow up questions were conducted through email communication. The final data from the interview is the complete transcription of the interview, which was then entered into HyperRESEARCH, a software for qualitative data analysis. 
DOI: https://doi.org/10.47405/aswj.v6i4.172

\section{Observation}

A second method of data collection that was proposed was to do an observation in either a classroom setting or workplace setting, depending on the participant's preference. However, this was modified due to feedback from the participant and this method would also be excluded in future designs. Additionally, the participant was not in a situation where a setting relevant for this study is available for observation. Feedback and discussion with the participant also revealed that observing potential participants may not be representative of the phenomenon. Given that the population for this study is small, it would not be possible to observe deaf individuals from Malaysia as a group. Observing a setting with only one deaf individual from Malaysia would be uncomfortable even though the observation is not intended to focus on the individual. Note that at the time that this research was conducted both the researcher and the participant were not located in Malaysia.

For this study, an alternative to observation in a real setting, was to observe a video recording of classroom setting that are available online, which unfortunately was also not easily available. Two videos portraying deaf students with their teachers were found and each video lasted for only a few minutes. The language and school uniform suggested they were elementary school students and teachers in Malaysia. It was noted that these videos were not enough to represent the actual phenomenon. Observation notes were prepared and entered into HyperRESEARCH for analysis.

\section{Document Reviews}

A third method of data collection was a document review. The researcher initially searched for media articles that had been published in Malaysia on any topic related to the deaf community. There were several media articles on the deaf community in Malaysia, however the researcher came across blog entries written by deaf individuals that were deemed more representative of this phenomenon. One article was identified on a blog of a deaf individual who attended a higher education institution in Malaysia. Permission was obtained from the author, and it was reviewed for this study. The document was reviewed, summarized, and entered into HyperRESEARCH for data analysis.

\section{Criteria for rigor}

Criteria for rigor that were established for this study include triangulation, member checking, peer debriefing and progressive subjectivity.

For internal validity, three different methods of data collection, which involved an interview, observation and document review were conducted. Data obtained from the three different resources did not contradict on another. It can be concluded that this study shows that the three resources were able to yield findings in a similar direction.

This research topic was developed based on the researcher's deep interest to contribute to the deaf community in Malaysia, therefore the researcher needed to establish that research analysis was not based on the researcher's own bias. Peer debriefing was conducted with an individual who was not involved with the data directly. The peer involved originates from Malaysia and was asked to review the codes and transcripts during the process of data analysis.

A third method for establishing rigor is through member checking. Member checking was conducted throughout the process of data collection, transcription, and data analysis. Transcripts and codes were presented to the participant for the participants' review and feedback.

A journal of personal development, which included emotions, opinions and reactions of the researcher, was maintained throughout the process of this research. This meets another criterion for rigor, which is progressive subjectivity. This allows the research to be consistently aware of any personal bias that can influence interpretation to the data. 
DOI: https://doi.org/10.47405/aswj.v6i4.172

\section{Result}

The interview session involved one participant and as this is a feasibility study, most of the background information is excluded from this report. The participant fulfilled all inclusion criteria to participate in this study. Specifically, the participant was originally from Malaysia and has had the experience of attending school and higher education institution in Malaysia. At the time of the research, the participant had been in the US for over 1 year and was attending a higher education institution in the US. Finally, the participant was a deaf individual above the age of 18 years old.

Data from all three methods of data collection were entered into HyperRESEARCH for analysis. Thematic analysis was performed. From this study, six main themes emerged.

\section{i. Access}

Based on data obtained from the interview, it is implied that access is likely an important factor for a deaf individual. Data suggest that having access to information or to activities that other hearing individuals can access is valued. It was deemed that when there is better access to information in general and a deaf individual can be more involved in activities, able to interact and engaged with people around them.

In Malaysia,

"I always saw many different clubs, but I only wish I knew who to contact or who to meet to join those clubs..."

In the United States,

"I can look for many things, you know who to contact, how to contact, how to access or join in. You can look for the information and ask around..."

\section{ii. Communication/interaction}

The theme of communication or interaction appeared several times in the data, which suggest that it is an important aspect of this phenomenon experienced by the participant and potentially other deaf individuals.

In Malaysia,

"The teachers were hearing, they could sign, but they were not really skilled signers. But it was okay, considerably good signing and teaching. They tried to teach, work and guide me and my friends."

"I tend to stay around the people I know, within my batch. This is the group of people that I study with, outside of this group, I didn't know anyone..."

"There are typically 50 to 150 students registered for a course. This therefore results in limited interaction between students and lecturers."

(document)

"Since I am not able to speak or lip-read, I have to depend on pen and paper as a mode of communication with others."

(document)

In the United States,

"I joined in with hearing people and it's fine. I meet and play (sports) with them, communication is not perfect but it's fine, I can play with them." 
DOI: https://doi.org/10.47405/aswj.v6i4.172

\section{iii.Gaining perspectives}

The emergence of this theme suggests that a deaf individual with this lived experience go through a change in perspective.

"By being here, has changed me, I feel that I've become a person who's willing to try, regardless I would go ahead and try things. If I feel there's something I want to try, I'd go ahead. I won't distant myself and not do anything about it, but I'll go ahead and give a try."

"If I have difficulty with a person, a hearing person, I will go ahead and talk about it with the person. Like here in (my institution), if anything happens, it's alright to speak with people. I learn how to go ahead and speak with people."

\section{iv. Interpreters}

The topic about availability of interpreters came up several times in the data collected. Data revealed that interpreters may not be easily available in Malaysia.

"No, I was on my own, there were no interpreters, and the lecturers didn't sign. But I asked the lecturers to write everything down on the board. I would try to read, take notes of the keywords, and try to understand the concepts. I did my own reading and writing. I'd ask questions, I'd ask the lecturers if I didn't understand."

"(In current institution) We have interpreters, so it's easy for me. I can take classes, sports, archery and join competitions..."

"As we know, local universities are currently not equipped with facilities/services for deaf students. The lecturers are not skilled in sign language and there are no interpreters. I have tried to request for interpreters, but they don't have financial allocations for interpreters. Furthermore, there aren't many interpreters in this country."

(document)

\section{v. Optimistic}

The data contained several descriptions that show optimism and how a deaf individual remains positive about their experiences.

"No, I was on my own, there were no interpreter, and the lecturers didn't sign. But I asked the lecturers to write everything down on the board. I would try to read, take notes of the key words, and try to understand the concept. I do my own reading and writing. I'd ask questions, I'd ask the lecturers if I don't understand. But that was okay...

I could do it. I didn't mind that the lecturers didn't sign, it didn't' matter. I could catch up fine."

"Based on everything that I've said, I felt that it was better for me to study in a local education institution, with hopes that I will be able to deal with the challenges that are to come and prove to the world, that deafness is not a hindrance for a deaf student from becoming educated such as encouraged by the prophet."

(document)

"Therefore, I have no choice but accept everything as the toughest challenge in my life."

(document) 
DOI: https://doi.org/10.47405/aswj.v6i4.172

\section{vi. Social Support}

Data revealed that positive and strong support from various people are important.

"Let me remember. I remember that after I got my results, a teacher told me, recommended me to apply to go to an institution. So, I did. I applied to several places, and I was accepted to (Malaysian institution). But they thought I was hearing. They didn't know that I was deaf. So, my parents and I went to discuss about it, discuss about me being deaf. They had no idea that I was deaf."

"Yes, because it would be difficult, I wouldn't know what to do, how to do, how to apply for a class. I didn't know that all, so I had to keep asking friends and they helped me. It helped a little, but it was limited information."

"Since I am the only deaf student on campus, a few of my friends have expressed interest in learning sign language. To date, I have a few friends who are partially skilled in sign language, and they are always helping me out throughout the course of this study"

(document)

\section{Discussion}

Given the sample size of this research, the emerging themes obtained from this study are limited only to the participant involved. However, these themes could be further explored to determine their significance. As an example, the theme "gaining perspectives" supports the conclusion made by De Clerk (2007) that such experience can have an impact on an individual's perspectives and worldview. Therefore, the findings of this feasibility study provide support for the researcher to continue exploring this topic.

Results obtained also revealed some of the factors that are worth exploring to further provide support for deaf community in Malaysia. Themes such as access, interaction/communication, interpreters are critical areas in the field of education for deaf students in general (Schick, Williams, \& Kupermintz, 2006). With this data, it suggests that those themes are also notable among the experience of deaf individuals in Malaysia. Further exploration on these areas can lead to findings that are significant and can be used to benefit deaf community in Malaysia.

Data also suggest that deaf individuals from Malaysia may have a positive outlook towards the many challenges they need to face. It is possible that there may be cultural values that are involved that become important factors contributing to the success of a deaf individuals in Malaysia. Data also revealed examples of the type of support that a deaf individual may receive from their family and people around them including friends and lecturers. This is not particularly surprising, given that a family unit is deemed as main source of support for many, specifically with regards to school or academic support (Othman, Azman, \& Mohd Ali ,2008).

With regards to the research methodology, various modifications were made to the methodology of this current study due to certain limitations. One of these limitations was limited number of deaf individuals who have experienced the phenomenon of interest. Hence, only one participant was recruited and participated in this current study. While it is a unique phenomenon, there is not enough participants to interview to further explore this specific phenomenon. The participant interviewed for this current study had a very specific and unique background that findings from a single case study may not be easily transferrable to the general population of deaf Malaysians, many of whom may not have the experience of living or attending school in Malaysia and in the United States.

The initial proposed study was designed to explore the general experience of living in Malaysia and in the United States including work and school. However, given that there was only one participant, and the participant's responses leaned more towards school experience, it was deemed appropriate to 


\section{Asian Social Work Journal (ASWJ), Volume 6, Issue 4, (page 1 - 9), 2021 DOI: https://doi.org/10.47405/aswj.v6i4.172}

narrow down the whole life experience to just school. Themes emerging from this study revealed that school experience alone has many facets and worthy of further explorations. Therefore, future studies may focus on exploring the school experience of deaf individuals in Malaysia.

Finally, this current study attempted a triangulation method including observations and document review. From this study, it was deemed that observations of deaf individuals in actual settings may not be appropriate. This is especially that the researcher was not located in Malaysia at the time of the research.

\section{Conclusion}

The results of this study provide support indicating that exploring the experience of deaf individuals can produce meaningful data that can be beneficial for the deaf community in the long run. It should be noted that this is a preliminary exploratory study. Findings reported from this study are not conclusive and are not generalizable. Studies in this area is still limited and therefore this current study will hopefully serve as a precursor to many more future studies.

\section{References}

De Clerck, G.A.M. (2007). Meeting Global Deaf Peers, Visiting Ideal Deaf Places: Deaf Ways of Education Leading to Empowerment, An Exploratory Case Study. American Annals of the Deaf 152(1): 5-19.

Evaer Video Recorder for Skype (Version 1.2.4.15) [Software]. Available from http://www.evaer.com/index.htm

Guba, E.G., \& Lincoln, Y.S. (1989). Fourth generation evaluation. Newbury Park, CA: Sage

HyperRESEARCH (Version 3.5.2) [Software]. Available from http://www.researchware.com/

Mertens, D.M. (2010). Research and evaluation in education and psychology. Thousand Oaks, CA: Sage

Othman, S.A., Azman, N. \& Mohd Ali, M. (2008). Faktor ibu bapa dalam kecemerlangan akademik pelajar pekak: Kajian kes retrospektif [Parent factor in academic excellence of deaf students: a retrospective case study]. Malaysian Journal of Learning and Instruction, 5, 79-97.

Schick, B., Williams, K., \& Kupermintz, H. (2006). Look who's being left behind: Educational interpreters and access to education for deaf and hard-of-hearing students. Journal of deaf studies and deaf education, 11(1), 3-20.

Schwandt, T.A. (2000). Three epistemological stances for qualitative inquiry: Interpretivism, hermeneutics, and social constructionism. In N.K. Denzin \& Y.S. Lincoln (Eds), Handbook of qualitative research ( $2^{\text {nd }}$ ed., pp. 189-214). Thousand Oaks, CA: Sage.

Wathum-Ocama, J.C. \& Rose, S. (2002). Hmong Immigrants' Views on the Education of their Deaf and Hard of Hearing Children. American Annals of the Deaf 147(3): 44-53

Wertz, F.J. (2011). A Phenomenological Psychological Approach to Trauma and Resilience. In F.J. Wertz, K. Charmaz, L.M. McMullen, R. Josselson, R. Anderson \& E. McSpadden (Eds.) Five Ways of Doing Qualitative Analysis (pp. 124-164). New York, NY: The Guilford Press.

Xue, M. (2013). Effects of Group Work on English Communicative Competence of Chinese International Graduates in United States Institutions of Higher Education. The Qualitative Report 18(4):1-19. 